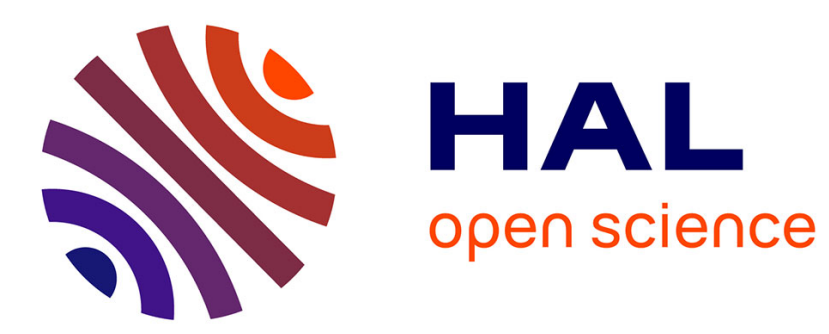

\title{
Dynamic substructuring of damped structures using singular value decomposition
}

Roger Ohayon, R. Sampaio, Christian Soize

\section{To cite this version:}

Roger Ohayon, R. Sampaio, Christian Soize. Dynamic substructuring of damped structures using singular value decomposition. Journal of Applied Mechanics, 1997, 64 (2), pp.292-298. 10.1115/1.2787306 . hal-00770023

\section{HAL Id: hal-00770023 \\ https://hal.science/hal-00770023}

Submitted on 4 Jan 2013

HAL is a multi-disciplinary open access archive for the deposit and dissemination of scientific research documents, whether they are published or not. The documents may come from teaching and research institutions in France or abroad, or from public or private research centers.
L'archive ouverte pluridisciplinaire HAL, est destinée au dépôt et à la diffusion de documents scientifiques de niveau recherche, publiés ou non, émanant des établissements d'enseignement et de recherche français ou étrangers, des laboratoires publics ou privés. 


\title{
Dynamic Substructuring of Damped Structures Using Singular Value Decomposition
}

\author{
R. Ohayon \\ Chair of Mechanics, CNAM, 2 rue Conté, F-75003 Paris, France (mem. ASME) \\ R. Sampaio \\ Department of Mechanical Engineering, Pontificia Universidade Católica do Rio de Janeiro, \\ 22453-900 Rio de Janeiro - RJ - Brazil \\ C. Soize \\ Structures Department, ONERA, BP 72, F-92322 Chatillon Cedex, France
}

\begin{abstract}
This paper deals with the theoretical aspects concerning linear elastodynamic of damped continuum medium in the frequency domain. Eigenvalue analysis and frequency response function are studied. The methods discussed here use a dynamic substructuring approach. The first method is based on a mixed variational formulation in which Lagrange multipliers are introduced to impose the linear constraints on the coupling interfaces. A modal reduction of each substructure is obtained using its free-interface modes. A practical construction of a unique solution is carried out using the Singular Value Decomposition (SVD) related only to the frequency-independent Lagrange multiplier terms. The second method is similar to the first one replacing the free-interface modes by the fixed-interface modes and elastostatic operator on the interface of each substructure.
\end{abstract}

\section{Introduction}

In this paper, we are interested in eigenvalue and frequency response function calculations of a linear dynamic three-dimensional bounded damped elastic structures subjected to prescribed forces. Recall that the frequency response functions allow deterministic and stationary random analyses to be performed (Kree and Soize, 1986; Argyris and Meljnek, 1991). More precisely, this paper is devoted to theoretical aspects of structure-structure coupling by dynamic substructuring methods using modal reduction procedures. The proposed methodology can be applied to general linear coupled systems such as fluid-structure interaction problems (Morand and Ohayon, 1995; Soize, Desanti and David, 1992).

For linear structural vibrations, dynamic substructuring techniques based on the use of the fixed-interface modes or free-interface modes (completed by static boundary functions, attachment modes, residual flexibility, etc.) of each substructure have been widely developed in the litterature: for conservative structures see for example (Hurty, 1965; Craig and Bampton, 1968; MacNeal, 1971; Rubin, 1975; Flashner, 1986; Min, Igusa and Achenbach, 1992; Farhat and Geradin, 1994) and for damped structures (Klein and Dowell, 1974; Hale and Meirovitch, 1980; Leung, 1993; Farstad and Singh, 1995; Rook and Singh, 1995).

Some papers are based on a mixed formulation using a Lagrange multiplier in order to impose the linear constraints on the coupling interfaces (see Klein and Dowell, 1974; Min, Igusa and Achenbach, 1992; Farstad and Singh, 1995; Rook and Singh, 1995). Within the context of finite element discretization of linear structural dynamic problems, Farhat and Geradin (1994) have also introduced a Lagrange multiplier to take into account incompatible meshes on the interface (their analysis is devoted to undamped structures using a component mode method based on fixedinterface modes and static boundary functions).

Below, we present an original general approach for damped structures using continuum-based variational formulations and Ritz-Galerkin projection methods using free-interface modes and fixed-interface modes of each substructure (in this paper we do not consider mathematical aspects of error estimates connected to the truncation of the modal series). For this purpose, various rigorous algebraic decompositions of admissible classes of the unknown fields are introduced and leads to several linear dynamic substructuring methods, the continuity of the displacement field on the interface being imposed through the use of a Lagrange multiplier field. As a consequence, the final system for the mixed formulation has a rank-deficiency in the matrix that describes the constraints. This leads to non-uniqueness of the solution. In order to avoid this difficulty, a new constructive approach is proposed consisting in using a Singular Value Decomposition (SVD) of the frequency-independent constraint matrix and chose a "least-square" solution that is in fact the solution of the original problem. Due to a relatively small number of degrees of freedom in the reduced model, the use of SVD is particularly efficient. Since the problem under consideration is linear, SVD is used only once. Consequently, the SVD appears as an efficient and reliable tool to solve this rank-deficiency problem. It should be noted that SVD has been used for undamped linear vibration analysis of plates using dynamic substructuring by analytical methods (Jen, Johnson and Dubois, 1995). Let us recall that SVD has also been used in the area of the nonlinear dynamical analysis of multibody systems with nonlinear constraints (Singh and Likins, 1985; Shabana , 1991; Schmidt and Müller, 1993).

Now we give a short description of the content of each section. Section 2 deals with the displacement and mixed variational formulations for the coupled linear structure-structure problem, Lagrange multiplier field being introduced in the mixed problem.

In Section 3, we present a dynamic substructuring method using the free-interface modes of each linear substructure. The modal reduction procedure is carried out using a new explicit construction of the Lagrange multiplier admissible space. Two practical constructions of the frequency response function of the global linear damped structure and the eigenvalues of the associated conservative structure are performed using SVD once on a part of the linear system to be solved, namely on the frequency-independent Lagrange multiplier terms.

Section 4 is devoted to a dynamic substructuring method using the classical Craig and Bampton fixed-interface modes and boundary static functions of each linear substructure, presented in an original general framework allowing various other decomposition procedures to be obtained. After having constructed the reduced matrix model of each substructure, we explain two procedures for the assemblage of the substructures and the construction of a solution, (1) in a classical manner and (2) as in Section 3 using Lagrange multiplier field and SVD.

Finally, in Section 5, some conclusions are presented. 


\section{Displacement and Mixed Variational For- mulations for the Coupled Structure-Structure Problem}

\subsection{General Mechanical Hypotheses}

In this section, the following hypotheses are introduced:

- One considers the linear vibrations of a three-dimensional structure about a static equilibrium configuration which is considered here as a natural state (for the sake of brevity, prestress are not considered but could be added without changing the theory).

- The structure is only submitted to prescribed external forces (no prescribed displacement).

With the above hypotheses, there are two cases.

(1)- The first one, which is the only case considered in this paper, corresponds to prescribed external forces which are in equilibrium at each instant. Consequently, the displacement field of the structure is defined up to an additive rigid body displacement field. In this case, we are only interested in the part of the displacement field due to the structural deformation. We will see below how the rigid body displacement field can be disregarded.

(2)- The second case corresponds to prescribed external forces which are not in equilibrium at some instants. To solve this problem, the method consists in transforming this case to the first case by adding an additional external force related to rigid body field. For the sake of brevity, this case will not be considered in the present paper.

One presents a variational formulation of the problem (first case), taking into account an additional small structural damping based on a linear viscoelastic model with an instantaneous memory. A frequency domain formulation is used, the convention for the Fourier transform being $\mathbf{u}(\omega)=\int_{\mathbb{R}} e^{-i \omega t} \mathbf{u}(t) d t$ where $\omega$ denotes the circular frequency, $\mathbf{u}(\omega)$ is a vector in $\mathbb{C}^{3}$ and $\overline{\mathbf{u}}(\omega)$ its conjugate ( $\mathbb{R}$ and $\mathbb{C}$ denote the set of real and complex numbers respectively).

\subsection{Notation for a Substructure $\Omega_{r}$}

We consider a structure formed by substructures that will be denoted by an index $r$. Let $\Omega_{r}$ be the 3D-bounded domain occupied at static equilibrium by the substructure labelled by index $r$. Let $\partial \Omega_{r}=\Gamma_{r} \cup \Gamma$ with $\Gamma_{r} \cap \Gamma=\emptyset$ be the boundary of $\Omega_{r}$ (assumed to be smooth). The boundary $\Gamma$ will be the interaction surface with another substructure. The external prescribed volumetric and surface force fields applied to $\Omega_{r}$ and $\Gamma_{r}$ are denoted by $\mathbf{g}_{\Omega_{r}}$ and $\mathbf{g}_{\Gamma_{r}}$ respectively. Let $\mathbf{u}^{r}=\left(u_{1}^{r}, u_{2}^{r}, u_{3}^{r}\right)$ be the displacement field at each point $\mathbf{x}=\left(x_{1}, x_{2}, x_{3}\right)$ in cartesian coordinates. The set of admissible displacement fields with values in $\mathbb{C}^{3}$ (resp. in $\mathbb{R}^{3}$ ) is denoted by $\mathcal{C}_{\Omega_{r}}$ (resp. $\mathcal{R}_{\Omega_{r}}$ ) and is used for dissipative problems (resp. associated conservative problems). For substructure $\Omega_{r}$, one denotes the test function (weighted function) associated with $\mathbf{u}^{r}$ as $\delta \mathbf{u}^{r} \in \mathcal{C}_{\Omega_{r}}$ (or in $\left.\mathcal{R}_{\Omega_{r}}\right)$. The strain tensor is defined by

$$
\varepsilon_{i j}\left(\mathbf{u}^{r}\right)=\frac{1}{2}\left(u_{i, j}^{r}+u_{j, i}^{r}\right),
$$

in which $v_{, j}$ denotes the partial derivative of $v$ with respect to $x_{j}$. The total stress tensor is defined by

$$
\sigma_{\mathrm{tot}}^{r}=\sigma^{r}+i \omega s^{r}
$$

where $\sigma^{r}$ is the elastic stress tensor defined by $\sigma_{i j}^{r}\left(\mathbf{u}^{r}\right)=a_{i j k h} \varepsilon_{k h}\left(\mathbf{u}^{r}\right)$ and $i \omega s^{r}$ is the viscous part of the total stress tensor such that $s_{i j}^{r}\left(\mathbf{u}^{r}\right)=$ $b_{i j k h} \varepsilon_{k h}\left(\mathbf{u}^{r}\right)$ (using summation over repeated indices). The mechanical coefficients $a_{i j k h}$ and $b_{i j k h}$ are independent of $\omega$ and verify the usual properties of symmetry and positivity (see Marsden and Hughes, 1983). The mass density is denoted by $\rho^{r}$. For the dissipative problem, three sesquilinear forms on $\mathcal{C}_{\Omega_{r}} \times \mathcal{C}_{\Omega_{r}}$ corresponding to the mass, stiffness and damping operators of substructure $\Omega_{r}$, are introduced as follows

$$
\begin{gathered}
m^{r}\left(\mathbf{u}^{r}, \delta \mathbf{u}^{r}\right)=\int_{\Omega_{r}} \rho^{r} \mathbf{u}^{r} \cdot \overline{\delta \mathbf{u}^{r}} d \mathbf{x} \\
k^{r}\left(\mathbf{u}^{r}, \delta \mathbf{u}^{r}\right)=\int_{\Omega_{r}} \sigma_{i j}^{r}\left(\mathbf{u}^{r}\right) \varepsilon_{i j}\left(\overline{\delta \mathbf{u}^{r}}\right) d \mathbf{x}, \\
d^{r}\left(\mathbf{u}^{r}, \delta \mathbf{u}^{r}\right)=\int_{\Omega_{r}} s_{i j}^{r}\left(\mathbf{u}^{r}\right) \varepsilon_{i j}\left(\overline{\delta \mathbf{u}^{r}}\right) d \mathbf{x} .
\end{gathered}
$$

It should be noted that the hermitian form $m^{r}$ is positive definite on $\mathcal{C}_{\Omega_{r}} \times \mathcal{C}_{\Omega_{r}}$. The hermitian forms $k^{r}$ and $d^{r}$ are semi-definite positive (degenerated forms) since rigid body displacement fields are allowed in the present case. The set $\mathcal{R}_{\text {rig }}^{r}$ of $\mathbb{R}^{3}$-valued rigid body displacement fields (of dimension 6) is a subset of $\mathcal{C}_{\Omega_{r}}$. Consequently, for all $\delta \mathbf{u}^{r}$ in $\mathcal{C}_{\Omega_{r}}$, $k^{r}\left(\mathbf{u}^{r}, \delta \mathbf{u}^{r}\right)$ and $d^{r}\left(\mathbf{u}^{r}, \delta \mathbf{u}^{r}\right)$ are equal to zero for any $\mathbf{u}^{r}$ in $\mathcal{R}_{\text {rig }}^{r}$. We then define the following sesquilinear form $z^{r}$ on $\mathcal{C}_{\Omega_{r}} \times \mathcal{C}_{\Omega_{r}}$

$$
z^{r}\left(\mathbf{u}^{r}, \delta \mathbf{u}^{r}\right)=-\omega^{2} m^{r}\left(\mathbf{u}^{r}, \delta \mathbf{u}^{r}\right)+i \omega d^{r}\left(\mathbf{u}^{r}, \delta \mathbf{u}^{r}\right)+k^{r}\left(\mathbf{u}^{r}, \delta \mathbf{u}^{r}\right)
$$

Finally, we define $\mathbf{f}^{r}$ by the relation

$$
\ll \mathbf{f}^{r}, \overline{\delta \mathbf{u}^{r}} \gg=\int_{\Omega_{r}} \mathbf{g}_{\Omega_{r}} \cdot \overline{\delta \mathbf{u}^{r}} d \mathbf{x}+\int_{\Gamma_{r}} \mathbf{g}_{\Gamma_{r}} \cdot \overline{\delta \mathbf{u}^{r}} d s .
$$

\subsection{Continuum-Based Variational Formulations for Two Coupled Substructures $\Omega_{1}$ and $\Omega_{2}$}

We consider a structure composed of two substructures $\Omega_{1}$ and $\Omega_{2}$ that interact through a common boundary $\Gamma$ (the extension to the case of more than two substructures is straightforward). The notations introduced in Section 2.2 are used with $r=1$ and $r=2$. The linear coupling conditions on $\Gamma$ are written as

$$
\begin{aligned}
\mathbf{u}^{1} & =\mathbf{u}^{2} \quad \text { on } \quad \Gamma, \\
\sigma_{\text {tot }}^{1} \mathbf{n}^{1} & =-\sigma_{\text {tot }}^{2} \mathbf{n}^{2} \quad \text { on } \quad \Gamma,
\end{aligned}
$$

where $\mathbf{n}^{r}$ is the unit normal to $\Gamma$, external to $\Omega^{r}$.

\subsubsection{Basic $\left(\mathbf{u}^{1}, \mathbf{u}^{2}\right)$ Variational Formulation $\mathcal{P}_{0}$}

For all real $\omega$ in $\mathbb{R}$ and prescribed $\left(\mathbf{f}^{1}, \mathbf{f}^{2}\right)$, find $\left(\mathbf{u}^{1}, \mathbf{u}^{2}\right)$ in $\mathcal{C}_{\Omega_{1}} \times \mathcal{C}_{\Omega_{2}}$ verifying the linear constraint $\mathbf{u}^{1}=\mathbf{u}^{2}$ on $\Gamma$, such that, for all $\left(\delta \mathbf{u}^{1}, \delta \mathbf{u}^{2}\right)$ in $\mathcal{C}_{\Omega_{1}} \times \mathcal{C}_{\Omega_{2}}$ verifying the linear constraint $\delta \mathbf{u}^{1}=\delta \mathbf{u}^{2}$ on $\Gamma$, one has

$$
z^{1}\left(\mathbf{u}^{1}, \delta \mathbf{u}^{1}\right)+z^{2}\left(\mathbf{u}^{2}, \delta \mathbf{u}^{2}\right)=\ll \mathbf{f}^{1}, \overline{\delta \mathbf{u}^{1}} \gg+\ll \mathbf{f}^{2}, \overline{\delta \mathbf{u}^{2}} \gg
$$

From the mathematical point of view (see Dautray and Lions, 1992), by taking Sobolev space $H^{1}\left(\Omega^{r}, \mathbb{C}^{3}\right)$ as admissible space $\mathcal{C}_{\Omega_{r}}$, the existence and uniqueness of a solution of $\mathcal{P}_{0}$ can be proved.

\subsubsection{Mixed $\left(\mathbf{u}^{1}, \mathbf{u}^{2}, \boldsymbol{\lambda}\right)$ Variational Formulation $\mathcal{P}_{1}$}

This formulation consists in relaxing the linear constraint (defined by Eq. (8)) used in $\mathcal{P}_{0}$ by the introduction of a Lagrange multiplier field $\boldsymbol{\lambda}$ defined on $\Gamma$. Let $\Lambda_{\Gamma}$ be the admissible set of Lagrange multiplier fields defined on $\Gamma$ with values in $\mathbb{C}^{3}$.

Formulation $\mathcal{P}_{1}$. For all real $\omega$ in $\mathbb{R}$ and prescribed $\left(\mathbf{f}^{1}, \mathbf{f}^{2}\right)$, find $\left(\mathbf{u}^{1}, \mathbf{u}^{2}\right)$ in $\mathcal{C}_{\Omega_{1}} \times \mathcal{C}_{\Omega_{2}}$ and $\boldsymbol{\lambda}$ in $\Lambda_{\Gamma}$ such that, for all $\left(\delta \mathbf{u}^{1}, \delta \mathbf{u}^{2}\right)$ in $\mathcal{C}_{\Omega_{1}} \times \mathcal{C}_{\Omega_{2}}$ and for all $\delta \boldsymbol{\lambda}$ in $\Lambda_{\Gamma}$, one has

$$
z^{1}\left(\mathbf{u}^{1}, \delta \mathbf{u}^{1}\right)+z^{2}\left(\mathbf{u}^{2}, \delta \mathbf{u}^{2}\right)+b\left(\boldsymbol{\lambda}, \delta \mathbf{u}^{1}-\delta \mathbf{u}^{2}\right)+b\left(\delta \boldsymbol{\lambda}, \mathbf{u}^{1}-\mathbf{u}^{2}\right)=\ll \mathbf{f}^{1}, \overline{\delta \mathbf{u}^{1}} \gg+\ll
$$

where $b(.$.$) is defined by$

$$
b\left(\boldsymbol{\lambda}, \mathbf{u}^{r}\right)=\int_{\Gamma} \boldsymbol{\lambda} \cdot \overline{\mathbf{u}^{r}} d s
$$


Space of Traces on $\Gamma$. The set of the traces related to the boundary $\Gamma$, is denoted by $\mathcal{C}_{\Gamma}$. Therefore, if $\mathbf{u}^{r} \in \mathcal{C}_{\Omega_{r}}$, then the trace of $\mathbf{u}^{r}$ on $\Gamma$ is denoted by $\mathbf{u}_{\mid \Gamma}^{r}$ and belongs to $\mathcal{C}_{\Gamma}$. In Eq. (11), $\Lambda_{\Gamma}$ is the dual space of $\mathcal{C}_{\Gamma}$.

Remark. From the mathematical point of view (see Dautray and Lions, 1992), by taking $\left.\mathcal{C}_{\Omega_{r}}=H^{1}\left(\Omega^{r}, \mathbb{C}^{3}\right)\right), \mathcal{C}_{\Gamma}=H^{1 / 2}\left(\Gamma, \mathbb{C}^{3}\right)$ and $\Lambda_{\Gamma}=H^{-1 / 2}\left(\Gamma, \mathbb{C}^{3}\right)$, the existence and uniqueness of a solution of formulation $\mathcal{P}_{1}$ can be proved using the so called LBB condition related to the sesquilinear form $b$ (see Brezzi and Fortin, 1991). It should be noted that $H^{1 / 2}\left(\Gamma, \mathbb{C}^{3}\right)$ is dense in $H^{-1 / 2}\left(\Gamma, \mathbb{C}^{3}\right)$.

\section{Dynamic Substructuring Using the Free-Inter- face Modes of Each Substructure}

The method is based on the use of the mixed variational formulation defined by $\mathcal{P}_{1}$. Then, a modal reduction is carried out using the RitzGalerkin projection on the free-interface modes of each substructure. Finally, the Singular Value Decomposition (SVD) is used for the construction of the solution.

\subsection{Free-Interface Modes of a Substructure $\Omega_{r}$}

A free-interface mode of a substructure $\Omega_{r}$ (for $\mathrm{r}=1$ or $\mathrm{r}=2$ ) is defined as an eigenmode of the conservative problem associated with the substructure $\Omega_{r}$, subject to zero forces on $\Gamma$. The real eigenvalues $\omega^{2} \geq 0$ and the eigenmodes $\mathbf{u}^{r}$ in $\mathcal{R}_{\Omega_{r}}$ are solutions of the following spectral problem: find $\omega^{2} \geq 0, \mathbf{u}^{r} \in \mathcal{R}_{\Omega_{r}}\left(\mathbf{u}^{r} \neq \mathbf{0}\right)$ such that for all $\delta \mathbf{u}^{r} \in \mathcal{R}_{\Omega_{r}}$, one has

$$
k^{r}\left(\mathbf{u}^{r}, \delta \mathbf{u}^{r}\right)=\omega^{2} m^{r}\left(\mathbf{u}^{r}, \delta \mathbf{u}^{r}\right)
$$

It can be shown that there exist six zero eigenvalues $0=\left(\omega_{-5}^{r}\right)^{2}=$ $\ldots=\left(\omega_{0}^{r}\right)^{2}$ (associated with the rigid body displacement fields) and that the strictly positive eigenvalues (associated with the displacement field due to structural deformation) constitute the increasing sequence $0<\left(\omega_{1}^{r}\right)^{2} \leq\left(\omega_{2}^{r}\right)^{2}, \ldots$. The six eigenvectors $\left\{\mathbf{u}_{-5}^{r}, \ldots, \mathbf{u}_{0}^{r}\right\}$ associated with zero eigenvalues span $\mathcal{R}_{\text {rig }}$ (space of the rigid body displacement fields). The family $\left\{\mathbf{u}_{-5}^{r}, \ldots, \mathbf{u}_{0}^{r} ; \mathbf{u}_{1}^{r}, \ldots\right\}$ of all the eigenvectors forms a complete set in $\mathcal{R}_{\Omega_{r}}$. For $\alpha$ and $\beta$ in $\{-5, \ldots, 0 ; 1, \ldots\}$, we have the orthogonality conditions

$$
\begin{gathered}
m^{r}\left(\mathbf{u}_{\alpha}^{r}, \mathbf{u}_{\beta}^{r}\right)=\delta_{\alpha \beta} \mu_{\alpha}^{r}, \\
k^{r}\left(\mathbf{u}_{\alpha}^{r}, \mathbf{u}_{\beta}^{r}\right)=\delta_{\alpha \beta} \mu_{\alpha}^{r} \omega_{\alpha}^{r 2},
\end{gathered}
$$

in which $\mu_{\alpha}^{r}>0$ is the generalized mass of mode $\alpha$ depending on the normalization of the eigenmodes.

\subsection{Modal Reduction of $\mathcal{P}_{1}$}

We introduce the subspace $\mathcal{C}_{\Omega_{r}}^{N_{r}}$ of $\mathcal{C}_{\Omega_{r}}$, of dimension $N_{r}$, spanned by $\left\{\mathbf{u}_{1}^{r}, \ldots, \mathbf{u}_{N_{r}}^{r}\right\}$ with $N_{r} \geq 1$. For all $\mathbf{u}^{r}$ in $\mathcal{C}_{\Omega_{r}}^{N_{r}}$, one has

$$
\mathbf{u}^{r}=\sum_{\alpha=1}^{N_{r}} q_{\alpha}^{r} \mathbf{u}_{\alpha}^{r}
$$

in which $q_{\alpha}^{r}$ are complex-valued generalized coordinates. Concerning the trace of the displacement field (including rigid body displacement field) on $\Gamma$, the subspace spanned by the family $\left\{\mathbf{u}_{-5 \mid \Gamma}^{r}, \ldots, \mathbf{u}_{0 \mid \Gamma}^{r} ; \mathbf{u}_{1 \mid \Gamma}^{r}, \ldots\right\}$ is a complete set in $\mathcal{C}_{\Gamma}$ (for the two domains $r=1$ and $r=2$ ). Consequently, the family $\left\{\mathbf{u}_{1 \mid \Gamma}^{r}, \ldots\right\}$ forms a complete set of the displacement field on $\Gamma$ due only to the structural deformation. Let $\mathcal{C}_{\Gamma}^{N_{r}}$ be the subspace of $\mathcal{C}_{\Gamma}$ spanned by the finite family $\left\{\mathbf{u}_{1 \mid \Gamma}^{r}, \ldots, \mathbf{u}_{N_{r} \mid \Gamma}^{r}\right\}$. Let $\mathcal{W}_{\Gamma}^{N}$ be the subspace of $\mathcal{C}_{\Gamma}$ of finite dimension $N \leq N_{1}+N_{2}$ defined by

$$
\mathcal{W}_{\Gamma}^{N}=\mathcal{C}_{\Gamma}^{N_{1}} \cup \mathcal{C}_{\Gamma}^{N_{2}}
$$

The present approach is based on the fact that any $\boldsymbol{\lambda}$ in $\Lambda_{\Gamma}$ can be expanded on a complete orthonormal set in $\mathcal{C}_{\Gamma}$ and consequently, the projection of the Lagrange multiplier $\boldsymbol{\lambda}$ is done on the subspace $\mathcal{W}_{\Gamma}^{N}$ of $\mathcal{C}_{\Gamma} \subset \Lambda_{\Gamma}$. A characterization of $\mathcal{W}_{\Gamma}^{N}$ requires the construction of a basis of $\mathcal{W}_{\Gamma}^{N}$ denoted by $\left\{\mathbf{w}_{1}, \ldots, \mathbf{w}_{N}\right\}$. One possible method consists in extracting an independent system of $N$ functions from the family $\left\{\mathbf{u}_{1 \mid \Gamma}^{1}, \ldots, \mathbf{u}_{N_{1 \mid \Gamma}}^{1}, \mathbf{u}_{1 \mid \Gamma}^{2}, \ldots, \mathbf{u}_{N_{2} \mid \Gamma}^{2}\right\}$. Consequently, for all $\boldsymbol{\lambda}$ in $\mathcal{W}_{\Gamma}^{N}$, one has

$$
\boldsymbol{\lambda}=\sum_{\gamma=1}^{N} p_{\gamma} \mathbf{w}_{\gamma}
$$

The Reduced Problem $\mathcal{P}_{1}^{\text {red }}$. We use the Ritz-Galerkin method consisting in substituting Eqs. (16) and (18) into Eq. (11). Using the orthogonality conditions defined by Eqs. (14) and (15) and introducing the vectors of generalized coordinates $\mathbf{q}^{1}=\left(q_{1}^{1}, \ldots, q_{N_{1}}^{1}\right), \mathbf{q}^{2}=\left(q_{1}^{2}, \ldots, q_{N_{2}}^{2}\right)$ and $\mathbf{p}=\left(p_{1}, \ldots, p_{N}\right)$, one deduces the following finite-dimension reduced problem from $\mathcal{P}_{1}$

$$
\left[\begin{array}{ccc}
\mathcal{Z}^{1}(\omega) & 0 & \mathcal{B}_{1}^{T} \\
0 & \mathcal{Z}^{2}(\omega) & \mathcal{B}_{2}^{T} \\
\mathcal{B}_{1} & \mathcal{B}_{2} & 0
\end{array}\right]\left[\begin{array}{c}
\mathbf{q}^{1} \\
\mathbf{q}^{2} \\
\mathbf{p}
\end{array}\right]=\left[\begin{array}{c}
\mathcal{F}^{1} \\
\mathcal{F}^{2} \\
\mathbf{0}
\end{array}\right]
$$

in which, for all real $\omega$ and for $r=1$ and $r=2,\left[\mathcal{Z}^{r}(\omega)\right]$ is an $\left(N_{r} \times\right.$ $\left.N_{r}\right)$ complex symmetric matrix, $\left[B_{r}\right]$ a $\left(N \times N_{r}\right)$ real matrix which is independent of $\omega$ and $\mathcal{F}^{r}$ a $\mathbb{C}^{N_{r}}$-valued vector. Matrix $\left[\mathcal{Z}^{r}(\omega)\right]$ is defined by

$$
\left[\mathcal{Z}^{r}(\omega)\right]=-\omega^{2}\left[\mathcal{M}^{r}\right]+i \omega\left[\mathcal{D}^{r}\right]+\left[\mathcal{K}^{r}\right],
$$

where $\left[\mathcal{M}^{r}\right]$ and $\left[\mathcal{K}^{r}\right]$ are diagonal positive-definite matrices such that $\left[\mathcal{M}^{r}\right]_{\alpha \beta}=\mu_{\alpha}^{r} \delta_{\alpha \beta}$ and $\left[\mathcal{K}^{r}\right]_{\alpha \beta}=\mu_{\alpha}^{r} \omega_{\alpha}^{r 2} \delta_{\alpha \beta},\left[\mathcal{D}^{r}\right]$ is a full symmetric positive-definite matrix, such that $\left[\mathcal{D}^{r}\right]_{\alpha \beta}=d^{r}\left(\mathbf{u}_{\beta}^{r}, \mathbf{u}_{\alpha}^{r}\right)$. Consequently, for all real $\omega$, matrix $\left[\mathcal{Z}^{r}(\omega)\right]$ is invertible. Matrix $\left[\mathcal{B}_{r}\right]$ is such that for all $\alpha$ in $\left\{1, \ldots, N_{r}\right\}$ and $\gamma$ in $\{1, \ldots, N\}$, one has

$$
\left[\mathcal{B}_{r}\right]_{\gamma \alpha}=b\left(\mathbf{w}_{\gamma}, \mathbf{u}_{\alpha}^{r}\right)
$$

Finally, vector $\mathcal{F}^{r}$ is such that, for all $\alpha$ in $\left\{1, \ldots, N_{r}\right\}$, one has

$$
\mathcal{F}_{\alpha}^{r}=\ll \mathbf{f}^{r}, \mathbf{u}_{\alpha}^{r} \gg
$$

3.3 Practical Construction of the Frequency Response Function of the Global Structure Using Reduced Problem $\mathcal{P}_{1}^{\text {red }}$ and SVD

First, we introduce the $(N \times M)$ real matrix $[\mathcal{B}]$ such that

$$
M=N_{1}+N_{2} \quad, \quad[\mathcal{B}]=\left[\begin{array}{ll}
\mathcal{B}_{1} & \mathcal{B}_{2}
\end{array}\right] .
$$

and write Eq. (19) as

$$
\left[\begin{array}{cc}
\mathcal{Z}(\omega) & \mathcal{B}^{T} \\
\mathcal{B} & 0
\end{array}\right]\left[\begin{array}{l}
\mathbf{q} \\
\mathbf{p}
\end{array}\right]=\left[\begin{array}{c}
\mathcal{F} \\
\mathbf{0}
\end{array}\right]
$$

In order to solve Eq. (24), we use a Singular Value Decomposition $(\mathrm{SVD})$ of $[\mathcal{B}]$. It is know that there exist algorithms (see Golub and Van Loan, 1989) which are very efficient for the construction of the SVD of reasonable size matrices. This is the case for the reduced problems obtained by modal projection as Eq. (24). In the proposed approach, it should be noted that SVD will only be applied to the submatrix $[\mathcal{B}]$ in Eq. (24). The SVD of $(N \times M)$ real matrix $[\mathcal{B}]$ with $M \geq N$ (see Section 3.2) consists in constructing the following decomposition

$$
[\mathcal{B}]=[U][\Sigma][V]^{T}
$$


where $[U]$ is an $(N \times N)$ orthogonal real matrix, $[V]$ is an $(M \times M)$ orthogonal real matrix and $[\Sigma]$ is a $(N \times M)$ real matrix which is written in block form as

$$
[\Sigma]=\left[\begin{array}{ll}
\Sigma^{+} & 0
\end{array}\right],
$$

in which [0] is the $(N \times(M-N))$ null matrix and $\left[\Sigma^{+}\right]$is the $(N \times N)$ diagonal matrix of positive or null singular values $\sigma_{k}$ such that $\sigma_{1} \geq$ $\sigma_{2} \geq \ldots \geq \sigma_{N} \geq 0$. Let $n$ be the integer such that $1 \leq n \leq N$ such that

$$
\sigma_{1} \geq \sigma_{2} \geq \ldots \geq \sigma_{n}>\sigma_{n+1}=\ldots=\sigma_{N}=0 .
$$

Consequently, the rank of $[\mathcal{B}]$ is equal to $n$ and Eq. (25) yields the SVD expansion

$$
[\mathcal{B}]=\sum_{k=1}^{n} \sigma_{k} \mathbf{U}^{k} \mathbf{V}^{k^{T}},
$$

in which the vectors $\mathbf{U}^{k}$ and $\mathbf{V}^{k}$ are the columns of $[U]$ and $[V]$ and such that

$$
<\mathbf{U}^{j}, \mathbf{U}^{k}>=\delta_{j k} \quad, \quad<\mathbf{V}^{j}, \mathbf{V}^{k}>=\delta_{j k} .
$$

The range of $[\mathcal{B}]$ is spanned by $\left\{\mathbf{U}^{1}, \ldots, \mathbf{U}^{N}\right\}$ and its null space by $\left\{\mathbf{V}^{n+1}, \ldots, \mathbf{V}^{M}\right\}$.

\subsubsection{First Algebraic Stage of the Practical Construction of Solution.}

En equation (24) has a unique solution if the null space of $[\mathcal{B}]^{T}$ is reduced to $\{0\}$ or equivalently, the dimension of the null space of $[\mathcal{B}]$ is equal to $M-N$, i.e. if one has $n=N$ in Eq. (27). Generally, we have $n<N$, which means that the linear constraint equations

$$
[\mathcal{B}] \mathbf{q}=0,
$$

are non independent and consequently, Eq. (24) does not have a unique solution. In that case, the SVD of $[\mathcal{B}]$ allows the construction of a unique solution $\mathbf{q}$ of Eq. (24) in the null space of $[\mathcal{B}]$, i.e.

$$
\mathbf{q}=\sum_{k=n+1}^{M} \xi_{k} \mathbf{V}^{k}
$$

Using Eqs. (28) and (29), it can be seen that $\mathbf{q}$ defined by Eq. (31) satisfies Eq. (30). Using Eqs. (28) and (31), Eq. (24) yields

$$
\sum_{k=n+1}^{M} \xi_{k}[\mathcal{Z}(\omega)] \mathbf{V}^{k}+\sum_{k=1}^{n} \sigma_{k} \eta_{k} \mathbf{V}^{k}=\mathcal{F}
$$

in which $\eta_{k}=<\mathbf{U}^{k}, \mathbf{p}>$, or equivalently,

$$
\sum_{k=n+1}^{M} \xi_{k} \mathbf{V}^{k}+\sum_{k=1}^{n} \sigma_{k} \eta_{k}[\mathcal{Z}(\omega)]^{-1} \mathbf{V}^{k}=[\mathcal{Z}(\omega)]^{-1} \mathcal{F}
$$

Equation (32) or (33) shows that $\xi_{k}$ can be calculated in a unique way.

3.3.2 Second Algebraic Stage of the Practical Construction of Solution.

First Procedure.

The projection of Eq. (33) on $\left\{\mathbf{V}^{1}, \ldots, \mathbf{V}^{n}\right\}$ yields

$$
[E(\omega)] \mathbf{y}=\mathbf{e}
$$

in which $[E(\omega)]$ is a $(n \times n)$ complex symmetric matrix such that $[E(\omega)]_{k^{\prime} k}$ $<[\mathcal{Z}(\omega)]^{-1} \mathbf{V}^{k}, \mathbf{V}^{k^{\prime}}>, \mathbf{y}=\left(y_{1}, \ldots, y_{n}\right)$ is a vector in $\mathbb{C}^{n}$ with $y_{k}=$ $\sigma_{k} \eta_{k}$ and $\mathbf{e}=\left(e_{1}, \ldots, e_{n}\right)$ is a vector in $\mathbb{C}^{n}$ such that
$e_{k}=<[\mathcal{Z}(\omega)]^{-1} \mathcal{F}, \mathbf{V}^{k}>$. Then, the projection of Eq. (33) on the remaining $\left\{\mathbf{V}^{n+1}, \ldots, \mathbf{V}^{M}\right\}$ yields for all $k$ in $\{n+1, \ldots, M\}$,

$$
\xi_{k}=-\sum_{k^{\prime}=1}^{n} y_{k^{\prime}}<[\mathcal{Z}(\omega)]^{-1} \mathbf{V}^{k^{\prime}}, \mathbf{V}^{k}>+<[\mathcal{Z}(\omega)]^{-1} \mathcal{F}, \mathbf{V}^{k}>
$$

The corresponding algorithm is summarized below.

Step 0: calculating the SVD of $[\mathcal{B}]$ in order to obtain its rank $n$ and $\mathbf{V}^{1}, \ldots, \mathbf{V}^{M}$.

Then, for each real $\omega$,

Step 1: solving the linear equation of dimension $n$ with $n+1$ right-hand side members $\left\{\mathcal{F} ; \mathbf{V}^{1}, \ldots, \mathbf{V}^{n}\right\}$

$$
[\mathcal{Z}(\omega)] \mathbf{X}^{0}=\mathcal{F} \quad ; \quad[\mathcal{Z}(\omega)] \mathbf{X}^{k}=\mathbf{V}^{k} \quad, \quad k \in\{1, \ldots, n\}
$$

Step 2: constructing $(n \times n)$ complex symmetric matrix $[E(\omega)]$ such that $[E(\omega)]_{k^{\prime} k}=<\mathbf{X}^{k}, \mathbf{V}^{k^{\prime}}>$ for $k$ and $k^{\prime}$ in $\{1, \ldots, n\}$;

Step 3: constructing $\mathbb{C}^{n}$-valued vector e such that $e_{k}=<\mathbf{X}^{0}, \mathbf{V}^{k}>$ for $k$ in $\{1, \ldots, n\}$;

Step 4: solving Eq. (34) which has a unique solution $\mathbf{y}$ (by construction); Step 5: calculating $\xi_{n+1}, \ldots, \xi_{M}$ such that for all $k$ in $\{n+1, \ldots, M\}$,

$$
\xi_{k}=-\sum_{k^{\prime}=1}^{n} y_{k^{\prime}}<\mathbf{X}^{k^{\prime}}, \mathbf{V}^{k}>+<\mathbf{X}^{0}, \mathbf{V}^{k}>.
$$

Step 6: calculating $\mathbf{q}$ by using Eq. (31).

\section{Second Procedure.}

The projection of Eq. (32) on $\left\{\mathbf{V}^{n+1}, \ldots, \mathbf{V}^{M}\right\}$ yields

$$
[G(\omega)] \boldsymbol{\xi}=\mathbf{g}
$$

in which $\boldsymbol{\xi}=\left(\xi_{n+1}, \ldots, \xi_{M}\right)$ is a vector in $\mathbb{C}^{M-n}, \mathbf{g}=\left(g_{1}, \ldots, g_{M-n}\right)$ is a vector in $\mathbb{C}^{M-n}$ such that $g_{k}=<\mathcal{F}, \mathbf{V}^{k+n}>$ and $[G(\omega)]$ is a $((M-n) \times(M-n))$ complex symmetric matrix such that

$$
[G(\omega)]=-\omega^{2}[\widehat{\mathcal{M}}]+i \omega[\widehat{\mathcal{D}}]+[\widehat{\mathcal{K}}]
$$

where $[\widehat{\mathcal{M}}],[\widehat{\mathcal{D}}]$ and $[\widehat{\mathcal{K}}]$ are $((M-n) \times(M-n))$ real symmetric positive-definite matrices defined, for all $k$ and $k^{\prime}$ in $\{1, \ldots, M-n\}$, by

$$
\begin{aligned}
& {[\widehat{\mathcal{M}}]_{k^{\prime} k}=<[\mathcal{M}] \mathbf{V}^{k+n}, \mathbf{V}^{k^{\prime}+n}>,} \\
& {[\widehat{\mathcal{D}}]_{k^{\prime} k}=<[\mathcal{D}] \mathbf{V}^{k+n}, \mathbf{V}^{k^{\prime}+n}>,} \\
& {[\widehat{\mathcal{K}}]_{k^{\prime} k}=<[\mathcal{K}] \mathbf{V}^{k+n}, \mathbf{V}^{k^{\prime}+n}>,}
\end{aligned}
$$

The corresponding algorithm is summarized below.

Step 0: calculating the SVD of $[\mathcal{B}]$ in order to obtain its rank $n$ and $\mathbf{V}^{n+1}, \ldots, \mathbf{V}^{M}$.

Then, for each real $\omega$,

Step 1: constructing $((M-n) \times(M-n))$ complex symmetric matrix $[G]$ such that

$[G]_{k^{\prime} k}=<[\mathcal{Z}(\omega)] \mathbf{V}^{k+n}, \mathbf{V}^{k^{\prime}+n}>$ for $k$ and $k^{\prime}$ in $\{1, \ldots, M-n\}$;

Step 2: constructing $\mathbb{C}^{M-n}$-valued vector $\mathbf{g}$ such that $g_{k}=\left\langle\mathcal{F}, \mathbf{V}^{k+n}\right\rangle$ ,$k \in\{1, \ldots, M-n\}$;

Step 3: solving Eq. (38) which has a unique solution $\boldsymbol{\xi}$ (by construction); Step 4: calculating $\mathbf{q}$ by using Eq. (31). 


\section{Comments on the two proposed procedures.}

(1)- Due to the fact that we have to solve a reduced size problem $N$ and $M$ are small.

(2)- In the first procedure, Step 1 is solved substructure by substructure independently. For each substructure $\Omega_{r}$, if the damping operator defined by Eq. (5) is diagonalized by the free-interface modes of this substructure, Step 1 is straightforward. If not, we have to solve a small $\left(N_{r} \times N_{r}\right)$ full complex symmetric system for each substructure. In Step 4, one has to solve a linear system of dimension $n$ with a full $(n \times n)$ complex symmetric matrix corresponding to the total number of independent linear constraints existing in the global structure (assemblage of all the substructures).

(3)- In the second procedure, Step1 is relative to the global structure (assemblage of all the substructures) and Step 4 requires to solve a full complex symmetric linear system of dimension $M-n$.

(4)- For example, if there are $N_{S}$ substructures (in this paper $N_{S}=2$ ) and if the mean value of $\left\{N_{r}\right\}$ on the set of substructures is $N_{R}=$ $\frac{1}{N_{S}} \sum_{r=1}^{N_{S}} N_{r}$, the order of floating operations is $N_{S} \times N_{R}^{3}$ for the first procedure with a damping matrix of each substructure which is not diagonalized by the free-interface modes of this substructure and, $N_{S}^{3} \times N_{R}^{4}$ for the second procedure.

As a conclusion, the first procedure is recommended since it is more efficient (particularly, if the damping matrix of each substructure is diagonalized by the free-interface modes of this substructure).

3.4 Practical Construction of the Eigenmodes of the Global Structure Using a Reduced Spectral Problem and SVD

The conservative problem associated to Eq. (24) leads to the following spectral problem

$$
\left[\begin{array}{ccc}
\mathcal{K}^{1} & 0 & \mathcal{B}_{1}^{T} \\
0 & \mathcal{K}^{2} & \mathcal{B}_{2}^{T} \\
\mathcal{B}_{1} & \mathcal{B}_{2} & 0
\end{array}\right]\left[\begin{array}{l}
\mathbf{q}^{1} \\
\mathbf{q}^{2} \\
\mathbf{p}
\end{array}\right]=\omega^{2}\left[\begin{array}{ccc}
\mathcal{M}^{1} & 0 & 0 \\
0 & \mathcal{M}^{2} & 0 \\
0 & 0 & 0
\end{array}\right]\left[\begin{array}{l}
\mathbf{q}^{1} \\
\mathbf{q}^{2} \\
\mathbf{p}
\end{array}\right],
$$

in which the two matrices defined by blocks are real symmetric and independent of $\omega$. Using a global notation as done in Eq. (24), Eq. (43) is rewritten as

$$
\left[\begin{array}{cc}
\mathcal{K} & \mathcal{B}^{T} \\
\mathcal{B} & 0
\end{array}\right]\left[\begin{array}{l}
\mathbf{q} \\
\mathbf{p}
\end{array}\right]=\omega^{2}\left[\begin{array}{cc}
\mathcal{M} & 0 \\
0 & 0
\end{array}\right]\left[\begin{array}{l}
\mathbf{q} \\
\mathbf{p}
\end{array}\right]
$$

For this problem, we must use the second procedure defined in Section 3.3.2 (in this case, the first procedure cannot be directly used since $[\mathcal{K}]-\omega^{2}[\mathcal{M}]$ is not invertible for all real values of $\left.\omega\right)$. Substituting Eq. (31) in the first row of Eq. (44), projecting it on $\left\{\mathbf{V}^{n+1}, \ldots, \mathbf{V}^{M}\right\}$ and using Eq. (29), yield

$$
[\widehat{\mathcal{K}}] \boldsymbol{\xi}=\omega^{2}[\widehat{\mathcal{M}}] \boldsymbol{\xi},
$$

in which $[\widehat{\mathcal{M}}]$ and $[\widehat{\mathcal{K}}]$ are defined by Eqs. (40) and (42).

The corresponding algorithm is summarized below.

Step 0: Calculating the SVD of $[\mathcal{B}]$ in order to obtain its rank $n$ and $\mathbf{V}^{n+1}, \ldots, \mathbf{V}^{M}$

Step 1: constructing $((M-n) \times(M-n))$ real symmetric matrices $[\widehat{\mathcal{M}}]$ and $[\widehat{\mathcal{K}}]$;

Step 2: solving the generalized eigenvalue problem defined by Eq. (45); Step 3: calculating the eigenmodes $\mathbf{u}=\left(\mathbf{u}^{1}, \mathbf{u}^{2}\right)$ of the structure by using Eqs. (31) and (16).

\section{Dynamic Substructuring Using the Fixed- Interface Modes of Each Substructure}

In this section, we present a modal reduction procedure based on formulation $\mathcal{P}_{1}$ using SVD (see Section 2.3.2) starting from a reduced matrix model for each substructure $\Omega_{r}$.

\subsection{Reduced Matrix Model of Substructure $\Omega_{r}$}

\subsubsection{Basic $\mathbf{u}^{r}$ Variational Formulation for Substructure $\Omega_{r}$}

Consider substructure $\Omega_{r}$ submitted to the external applied forces $\mathbf{g}_{\Omega_{r}}$ in $\Omega_{r}, \mathbf{g}_{\Gamma_{r}}$ on $\Gamma_{r}$ and $\mathbf{g}_{\Gamma}$ on the interaction surface $\Gamma$.

The basic variational formulation for substructure $\Omega_{r}$ is written as follows.

Basic problem $\mathcal{P}_{1}^{r}$. For all real $\omega$ in $\mathbb{R}$ and prescribed $\mathbf{f}^{r}$ defined by Eq. (7), find $\mathbf{u}^{r}$ in $\mathcal{C}_{\Omega_{r}}$ such that, for all $\delta \mathbf{u}^{r}$ in $\mathcal{C}_{\Omega_{r}}$, one has

$$
z^{r}\left(\mathbf{u}^{r}, \delta \mathbf{u}^{r}\right)=\ll \mathbf{f}^{r}, \overline{\delta \mathbf{u}^{r}} \gg+\ll \mathbf{f}_{\Gamma}, \overline{\delta \mathbf{u}^{r}} \gg
$$

in which $z^{r}$ is defined by Eq. (6) and where $\ll \mathbf{f}_{\Gamma}, \overline{\delta \mathbf{u}^{r}} \gg=\int_{\Gamma} \mathbf{g}_{\Gamma} \cdot \overline{\delta \mathbf{u}^{r}} d s$.

\subsubsection{Fixed-Interface Modes of Substructure $\Omega_{r}$}

A fixed-interface mode of a substructure $\Omega_{r}$ (for $\mathrm{r}=1$ or $\mathrm{r}=2$ ) is defined as an eigenmode of the conservative problem associated with the substructure $\Omega_{r}$, which is fixed on $\Gamma$. Since the problem is conservative and defined in a bounded domain, all the quantities are real. Consequently, we introduce the set $\mathcal{R}_{\Omega_{r}}^{0}$ defined by

$$
\mathcal{R}_{\Omega_{r}}^{0}=\left\{\delta \mathbf{u}^{r} \in \mathcal{R}_{\Omega_{r}} \mid \delta \mathbf{u}^{r}=\mathbf{0} \quad \text { on } \quad \Gamma\right\},
$$

in which $\mathcal{R}_{\Omega_{r}}$ is defined in Section 2.2. The real eigenvalues $\omega^{2}>0$ and the eigenmodes $\mathbf{u}^{r}$ in $\mathcal{R}_{\Omega_{r}}^{0}$ are solution of the following spectral problem: Find $\omega^{2}>0, \mathbf{u}^{r} \in \mathcal{R}_{\Omega_{r}}^{0}\left(\mathbf{u}^{r} \neq \mathbf{0}\right)$ such that for all $\delta \mathbf{u}^{r} \in \mathcal{R}_{\Omega_{r}}^{0}$, one has

$$
k^{r}\left(\mathbf{u}^{r}, \delta \mathbf{u}^{r}\right)=\omega^{2} m^{r}\left(\mathbf{u}^{r}, \delta \mathbf{u}^{r}\right),
$$

in which $m^{r}$ and $k^{r}$ are defined by Eqs. (3) and (4) respectively. It can be shown that the eigenvalues constitute and increasing sequence $0<\left(\omega_{1}^{r}\right)^{2} \leq\left(\omega_{2}^{r}\right)^{2}, \ldots$. The family $\left\{\mathbf{u}_{1}^{r}, \mathbf{u}_{2}^{r}, \ldots\right\}$ of the eigenvectors associated with the eigenvalues, forms a complete set in $\mathcal{R}_{\Omega_{r}}^{0}$. For $\alpha$ and $\beta$ in $\{1,2, \ldots\}$, we have the orthogonality conditions similar to Eqs. (14) and (15).

\subsubsection{Introduction of the Elastostatic Lifting Operator $S^{r}$}

We consider the solution $\mathbf{u}_{\text {stat }}^{r}$ of the elastostatic problem of substructure $\Omega_{r}$ subjected to a prescribed displacement field $\mathbf{u}_{\mid \Gamma}^{r}$ on $\Gamma$. Let $\mathcal{R}_{\Gamma}$ and $\mathcal{R}_{\Omega_{r}}^{\mathbf{u}_{\mid \Gamma}^{r}}$ be the sets of functions such that

$$
\begin{gathered}
\mathcal{R}_{\Gamma}=\left\{\mathbf{x} \mapsto \mathbf{u}_{\Gamma}(\mathbf{x}) \quad, \quad \forall \mathbf{x} \in \Gamma\right\} \quad, \\
\mathcal{R}_{\Omega_{r}}^{\mathbf{u}_{\mid \Gamma}^{r}}=\left\{\mathbf{u}^{r} \in \mathcal{R}_{\Omega_{r}} \mid \quad \mathbf{u}^{r}=\mathbf{u}_{\mid \Gamma}^{r} \quad \text { on } \quad \Gamma\right\} .
\end{gathered}
$$

The field $\mathbf{u}_{\text {stat }}^{r}$ satisfies the following variational formulation

$$
k^{r}\left(\mathbf{u}_{\text {stat }}^{r}, \delta \mathbf{u}^{r}\right)=0 \quad, \quad \mathbf{u}_{\text {stat }}^{r} \in \mathcal{R}_{\Omega_{r}}^{\mathbf{u}_{\mid \Gamma}^{r}} \quad, \quad \forall \delta \mathbf{u}^{r} \in \mathcal{R}_{\Omega_{r}}^{0},
$$

where $\mathcal{R}_{\Omega_{r}}^{0}$ is the space $\mathcal{R}_{\Omega_{r}}^{\mathbf{u}_{\mid \Gamma}^{r}}$ obtained for $\mathbf{u}_{\mid \Gamma}^{r}=\mathbf{0}$. The solution $\mathbf{u}_{\text {stat }}^{r}$ of Eq. (51) defines the linear operator $S^{r}$ from $\mathcal{R}_{\Gamma}$ into $\mathcal{R}_{\Omega_{r}}$ (called lifting operator in mathematics), such that

$$
\mathbf{u}_{\mid \Gamma}^{r} \mapsto \mathbf{u}_{\text {stat }}^{r}=S^{r}\left(\mathbf{u}_{\mid \Gamma}^{r}\right)
$$

We denote the range space of operator $S^{r}$ as $\mathcal{R}_{\Omega_{r}}^{\Gamma} \subset \mathcal{R}_{\Omega_{r}}$ such that $\mathcal{R}_{\Omega_{r}}^{\Gamma}=S^{r}\left(\mathcal{R}_{\Gamma}\right)$. It should be noted that the discretization of $S^{r}$ by the finite element method is obtained by a classical static condensation procedure (sometimes called the Schur complement) of the stiffness matrix of substructure $\Omega_{r}$ with respect to degrees of freedom on $\Gamma$. 


\subsubsection{Conjugate Relationships Between $\mathbf{u}_{\alpha}^{r}$ and $\mathbf{u}_{\text {stat }}^{r}$}

Taking $\delta \mathbf{u}^{r}=\mathbf{u}_{\alpha}^{r}$ in Eq. (51), for $\mathbf{u}_{\text {stat }}^{r}$ satisfying Eq. (51) yields

$$
k^{r}\left(\mathbf{u}_{\text {stat }}^{r}, \mathbf{u}_{\alpha}^{r}\right)=0
$$

For a given mode $\left(\omega_{\alpha}^{r}, \mathbf{u}_{\alpha}^{r} \in \mathcal{R}_{\Omega_{r}}^{0}\right)$, the modal reaction forces $\mathbf{F}_{\alpha}^{r}=$ $\sigma^{r}\left(\mathbf{u}_{\alpha}^{r}\right) \mathbf{n}^{r}$ on $\Gamma$ is defined by the variational property

$$
k^{r}\left(\mathbf{u}_{\alpha}^{r}, \delta \mathbf{u}^{r}\right)-\left(\omega_{\alpha}^{r}\right)^{2} m^{r}\left(\mathbf{u}_{\alpha}^{r}, \delta \mathbf{u}^{r}\right)=\int_{\Gamma} \mathbf{F}_{\alpha}^{r} \cdot \delta \mathbf{u}^{r} d s, \forall \delta \mathbf{u}^{r} \in \mathcal{R}_{\Omega_{r}} .
$$

Using Eqs. (48) and (53), Eq. (54) yields

$$
m^{r}\left(\mathbf{u}_{\text {stat }}^{r}, \mathbf{u}_{\alpha}^{r}\right)=-\frac{1}{\left(\omega_{\alpha}^{r}\right)^{2}} \int_{\Gamma} \mathbf{F}_{\alpha}^{r} \cdot \mathbf{u}_{\mid \Gamma}^{r} d s
$$

Consequently, for all field $\mathbf{u}_{\mid \Gamma}^{r}$ in $\mathcal{R}_{\Gamma}$ and $\mathbf{u}_{\alpha}^{r}$ in $\mathcal{R}_{\Omega_{r}}^{0}$, one has

$$
\begin{gathered}
k^{r}\left(S^{r}\left(\mathbf{u}_{\mid \Gamma}^{r}\right), \mathbf{u}_{\alpha}^{r}\right)=0 \\
m^{r}\left(S^{r}\left(\mathbf{u}_{\mid \Gamma}^{r}\right), \mathbf{u}_{\alpha}^{r}\right)=-\frac{1}{\left(\omega_{\alpha}^{r}\right)^{2}} \int_{\Gamma} \mathbf{F}_{\alpha}^{r} \cdot \mathbf{u}_{\mid \Gamma}^{r} d s
\end{gathered}
$$

4.1.5 Decomposition of $\mathcal{R}_{\Omega_{r}}$ and $\mathcal{C}_{\Omega_{r}}$

Due to the fact that the trace of $\mathbf{u}^{r}-\mathbf{u}_{\text {stat }}^{r}$ is zero on $\Gamma$, we have the following decomposition

$$
\begin{gathered}
\mathcal{R}_{\Omega_{r}}=\mathcal{R}_{\Omega_{r}}^{\Gamma} \oplus \mathcal{R}_{\Omega_{r}}^{0}, \\
\mathbf{u}^{r}=S^{r}\left(\mathbf{u}_{\mid \Gamma}^{r}\right)+\sum_{\alpha=1}^{\infty} q_{\alpha}^{r} \mathbf{u}_{\alpha}^{r} .
\end{gathered}
$$

Let $\mathcal{C}_{\Omega_{r}}^{\Gamma}$ and $\mathcal{C}_{\Omega_{r}}^{0}$ be the complexified vector spaces of $\mathcal{R}_{\Omega_{r}}^{\Gamma}$ and $\mathcal{R}_{\Omega_{r}}^{0}$ respectively. One then has

$$
\mathcal{C}_{\Omega_{r}}=\mathcal{C}_{\Omega_{r}}^{\Gamma} \oplus \mathcal{C}_{\Omega_{r}}^{0}
$$

and Eq. (59) holds with $\mathbf{u}_{\mid \Gamma}^{r}$ being a $\mathbb{C}^{3}$-valued field and $q_{\alpha}^{r}$ complex numbers.

\subsubsection{Construction of the Reduced Matrix Model}

We introduce the subspace $\mathcal{C}_{\Omega_{r}}^{0, N_{r}}$ of $\mathcal{C}_{\Omega_{r}}^{0}$, of dimension $N_{r}$, spanned by $\left\{\mathbf{u}_{1}^{r}, \ldots, \mathbf{u}_{N_{r}}^{r}\right\}$ with $N_{r} \geq 1$ and the subspace $\mathcal{C}_{\Omega_{r}}^{\Gamma, N_{r}}$ of $\mathcal{C}_{\Omega_{r}}$ such that

$$
\mathcal{C}_{\Omega_{r}}^{\Gamma, N_{r}}=\mathcal{C}_{\Omega_{r}}^{\Gamma} \oplus \mathcal{C}_{\Omega_{r}}^{0, N_{r}}
$$

For all $\mathbf{u}^{r}$ and $\delta \mathbf{u}^{r}$ in $\mathcal{C}_{\Omega_{r}}^{N_{r}}$, one has

$$
\begin{gathered}
\mathbf{u}^{r}=S^{r}\left(\mathbf{u}_{\mid \Gamma}^{r}\right)+\sum_{\alpha=1}^{N_{r}} q_{\alpha}^{r} \mathbf{u}_{\alpha}^{r}, \\
\delta \mathbf{u}^{r}=S^{r}\left(\delta \mathbf{u}_{\mid \Gamma}^{r}\right)+\sum_{\alpha=1}^{N_{r}} \delta q_{\alpha}^{r} \mathbf{u}_{\alpha}^{r} .
\end{gathered}
$$

We use the Ritz-Galerkin method related to space $\mathcal{C}_{\Omega_{r}}^{0}$ consisting in substituting Eqs. (62) and (63) into Eq. (46). Using the conjugate relations (56) and (57) and the orthogonality properties (14) and (15) for fixed-interface modes, we obtain in abstract operator notation

$$
\left[\begin{array}{cc}
\mathbf{Z}_{\Gamma}^{r}(\omega) & { }^{t} \mathcal{A}^{r}(\omega) \\
\mathcal{A}^{r}(\omega) & {\left[\mathcal{Z}^{r}(\omega)\right.}
\end{array}\right]\left[\begin{array}{l}
\mathbf{u}_{\Gamma}^{r} \\
\mathbf{q}^{r}
\end{array}\right]=\left[\begin{array}{l}
\mathbf{f}_{\Gamma} \\
\mathcal{F}^{r}
\end{array}\right]
$$

in which $\mathbf{q}^{r}=\left(q_{1}^{r}, \ldots, q_{N_{r}}^{r}\right)$ is the vector of generalized coordinates related to the fixed-interface modes, $\mathcal{F}^{r}=\left(\mathcal{F}_{1}, \ldots, \mathcal{F}_{N_{r}}\right)$ is the vector whose components are given by Eq. (22) using the fixed-interface modes and $\mathbf{f}_{\Gamma}$ is defined in Section 4.1.1.

(1)- For all real $\omega$, linear operator $\mathbf{Z}_{\Gamma}^{r}(\omega)$ is defined by the following sesquilinear form on $\mathcal{C}_{\Omega_{r}}^{\Gamma} \times \mathcal{C}_{\Omega_{r}}^{\Gamma}$

$$
\ll \mathbf{Z}_{\Gamma}^{r}(\omega) \mathbf{u}_{\mid \Gamma}^{r}, \overline{\delta \mathbf{u}_{\mid \Gamma}^{r}} \gg=z^{r}\left(S^{r}\left(\mathbf{u}_{\mid \Gamma}^{r}\right), S^{r}\left(\delta \mathbf{u}_{\mid \Gamma}^{r}\right)\right) .
$$

From Eq. (6), we deduce the following abstract operator equation

$$
\mathbf{Z}_{\Gamma}^{r}(\omega)=-\omega^{2} \mathbf{M}_{\Gamma}^{r}+i \omega \mathbf{D}_{\Gamma}^{r}+\mathbf{K}_{\Gamma}^{r},
$$

in which the mass, damping and stiffness operators $\mathbf{M}_{\Gamma}^{r}, \mathbf{D}_{\Gamma}^{r}$ and $\mathbf{K}_{\Gamma}^{r}$ are defined by

$$
\begin{aligned}
& \ll \mathbf{M}_{\Gamma}^{r} \mathbf{u}_{\mid \Gamma}^{r}, \overline{\delta \mathbf{u}_{\mid \Gamma}^{r}} \gg=m^{r}\left(S^{r}\left(\mathbf{u}_{\mid \Gamma}^{r}\right), S^{r}\left(\delta \mathbf{u}_{\mid \Gamma}^{r}\right)\right), \\
& \ll \mathbf{D}_{\Gamma}^{r} \mathbf{u}_{\mid \Gamma}^{r}, \overline{\delta \mathbf{u}_{\mid \Gamma}^{r}} \gg=d^{r}\left(S^{r}\left(\mathbf{u}_{\mid \Gamma}^{r}\right), S^{r}\left(\delta \mathbf{u}_{\mid \Gamma}^{r}\right)\right), \\
& \ll \mathbf{K}_{\Gamma}^{r} \mathbf{u}_{\mid \Gamma}^{r}, \overline{\delta \mathbf{u}_{\mid \Gamma}^{r}} \gg=k^{r}\left(S^{r}\left(\mathbf{u}_{\mid \Gamma}^{r}\right), S^{r}\left(\delta \mathbf{u}_{\mid \Gamma}^{r}\right)\right),
\end{aligned}
$$

where $m^{r}, k^{r}$ and $d^{r}$ are defined by Eqs. (3), (4) and (5), respectively. It should be noted that these operators are related to surface $\Gamma$ and correspond to the static condensation on $\Gamma$ of the mass, stiffness (Guyan, 1965) and damping operators using the elastostatic operator $S^{r}$ defined in Section 4.1.3.

(2)- For all real $\omega$, the $\left(N_{r} \times N_{r}\right)$ complex symmetric matrix $\left[\mathcal{Z}^{r}(\omega)\right]$ is defined by Eq. (20) using the fixed-interface modes. It should be noted that if the damping operator defined by Eq. (5) is diagonalized by the fixed-interface modes, matrix $\left[\mathcal{Z}^{r}(\omega)\right]$ is diagonal.

(3)- For all real $\omega$, the linear operator $\mathcal{A}^{r}(\omega)$ is defined by the following sesquilinear form on $\mathcal{C}_{\Omega_{r}}^{\Gamma} \times \mathbb{C}^{N_{r}}$

$$
\ll \mathcal{A}^{r}(\omega) \mathbf{u}_{\mid \Gamma}^{r}, \overline{\delta \mathbf{q}^{r}} \gg=\sum_{\alpha=1}^{N_{r}} z^{r}\left(S^{r}\left(\mathbf{u}_{\mid \Gamma}^{r}\right), \mathbf{u}_{\alpha}^{r}\right) \overline{\delta q_{\alpha}^{r}},
$$

in which $\delta \mathbf{q}^{r}=\left(\delta q_{1}^{r}, \ldots, \delta q_{N_{r}}^{r}\right)$. From Eq. (6), we deduce the following abstract operator equation

$$
\mathcal{A}^{r}(\omega)=-\omega^{2} \mathcal{A}_{m}^{r}+i \omega \mathcal{A}_{d}^{r}
$$

in which $\mathcal{A}_{m}^{r}$ and $\mathcal{A}_{d}^{r}$ are operators defined by

$$
\begin{aligned}
& \ll \mathcal{A}_{m}^{r} \mathbf{u}_{\mid \Gamma}^{r}, \overline{\delta \mathbf{q}^{r}} \gg=\sum_{\alpha=1}^{N_{r}} m^{r}\left(S^{r}\left(\mathbf{u}_{\mid \Gamma}^{r}\right), \mathbf{u}_{\alpha}^{r}\right) \overline{\delta q_{\alpha}^{r}}, \\
& \ll \mathcal{A}_{d}^{r} \mathbf{u}_{\mid \Gamma}^{r}, \overline{\delta \mathbf{q}^{r}} \gg=\sum_{\alpha=1}^{N_{r}} d^{r}\left(S^{r}\left(\mathbf{u}_{\mid \Gamma}^{r}\right), \mathbf{u}_{\alpha}^{r}\right) \overline{\delta q_{\alpha}^{r}}
\end{aligned}
$$

in which Eq. (56) has been used. The quantities $m^{r}\left(S^{r}\left(\mathbf{u}_{\mid \Gamma}^{r}\right), \mathbf{u}_{\alpha}^{r}\right)$ are calculated using Eq. (57) and $d^{r}\left(S^{r}\left(\mathbf{u}_{\mid \Gamma}^{r}\right), \mathbf{u}_{\alpha}^{r}\right)$ using Eqs. (5), (51) and (52). Finally, operator ${ }^{t} \mathcal{A}^{r}(\omega)$ is defined by the following sesquilinear form on $\mathbb{C}^{N_{r}} \times \mathcal{C}_{\Omega_{r}}^{\Gamma}$ such that

$$
\ll^{t} \mathcal{A}^{r}(\omega) \mathbf{q}^{r}, \overline{\delta \mathbf{u}_{\mid \Gamma}^{r}} \gg=\sum_{\alpha=1}^{N_{r}} q_{\alpha}^{r} z^{r}\left(\mathbf{u}_{\alpha}^{r}, S^{r}\left(\delta \mathbf{u}_{\mid \Gamma}^{r}\right)\right) .
$$

In conclusion, the matrix (of operators) in the left-hand side of Eq. (64) is called the "reduced matrix model" of substructure $\Omega_{r}$ relative to the displacement field $\mathbf{u}_{\mid \Gamma}^{r}$ on $\Gamma$ and the $N_{r}$ generalized coordinates (which can be viewed as "internal generalized degrees of freedom"). We refer to Morand and Ohayon (1995) for the particular case of an undamped structure. 
4.2 Frequency Response Function and Eigenmodes Constructions for the Global Structure Using the Mixed Variational Formulation and SVD

\subsubsection{Modal Reduction of Mixed Problem $\mathcal{P}_{1}$}

The reduction of $\mathcal{P}_{1}$ defined in Section 2.3.2 is obtained using the reduced matrix model defined by Eq. (64) for each substructure. Recall that the projection of Lagrange multiplier $\boldsymbol{\lambda}$ must be done on the subspace $\mathcal{W}_{\Gamma}^{N}$ of $\mathcal{C}_{\Gamma} \subset \Lambda_{\Gamma}$. A characterization of $\mathcal{W}_{\Gamma}^{N}$ requires the construction of a basis of $\mathcal{W}_{\Gamma}^{N}$ denoted by $\left\{\mathbf{w}_{1}, \ldots, \mathbf{w}_{N}\right\}$. Consequently, for all $\boldsymbol{\lambda}$ in $\mathcal{W}_{\Gamma}^{N}$, one has Eq. (18). Substituting Eqs. (62),(63), (18) and $\delta \boldsymbol{\lambda}=\sum_{\gamma=1}^{N} \delta p_{\gamma} \mathbf{w}_{\gamma}$ into Eq. (11), we obtain

$$
\left[\begin{array}{ccccc}
\mathbf{Z}_{\Gamma}^{1}(\omega) & { }^{t} \mathcal{A}^{1}(\omega) & \mathbf{0} & \mathbf{0} & { }^{t} \mathcal{B}_{1} \\
\mathcal{A}^{1}(\omega) & {\left[\mathcal{Z}^{1}(\omega)\right]} & \mathbf{0} & 0 & 0 \\
\mathbf{0} & \mathbf{0} & \mathbf{Z}_{\Gamma}^{2}(\omega) & { }^{t} \mathcal{A}^{2}(\omega) & { }^{t} \mathcal{B}_{2} \\
\mathbf{0} & 0 & \mathcal{A}^{2}(\omega) & {\left[\mathcal{Z}^{2}(\omega)\right]} & 0 \\
\mathcal{B}_{1} & 0 & \mathcal{B}_{2} & 0 & 0
\end{array}\right]\left[\begin{array}{c}
\mathbf{u}_{\Gamma}^{1} \\
\mathbf{q}^{1} \\
\mathbf{u}_{\Gamma}^{2} \\
\mathbf{q}^{2} \\
\mathbf{p}
\end{array}\right]=\left[\begin{array}{c}
\mathbf{0} \\
\mathcal{F}^{1} \\
\mathbf{0} \\
\mathcal{F}^{2} \\
0
\end{array}\right]
$$

in which we can recognize the reduced model of each substructure (see Eq. (64)). Using Eq. (12), for $r=1,2$ and $\gamma$ in $\{1, \ldots, N\}$, operators $\mathcal{B}_{1}$ and $\mathcal{B}_{2}$ are defined by

$$
\left[\mathcal{B}_{r}\right]_{\gamma}=b\left(\mathbf{w}_{\gamma}, \mathbf{u}_{\Gamma}^{r}\right)
$$

4.2.2 Practical Construction of the Frequency Response Function Using SVD

Since $\mathcal{B}_{1}$ and $\mathcal{B}_{2}$ are independent of $\omega$, Eq. (75) can be rewritten as

$$
\left[\begin{array}{cc}
\mathcal{Z}(\omega) & { }^{t} \mathcal{B} \\
\mathcal{B} & 0
\end{array}\right]\left[\begin{array}{l}
\mathbf{Q} \\
\mathbf{p}
\end{array}\right]=\left[\begin{array}{l}
\mathcal{F} \\
\mathbf{0}
\end{array}\right]
$$

where $\mathbf{Q}=\left(\mathbf{u}_{\Gamma}^{1}, \mathbf{q}^{1}, \mathbf{u}_{\Gamma}^{2}, \mathbf{q}^{2}\right)$. Equation (77) being similar to Eq. (24), the practical construction is carried out as described in Section 3.3.

\subsubsection{Practical Construction of the Eigenmodes Using SVD}

The conservative problem associated to Eq. (75) leads to the following spectral problem

$$
\begin{array}{r}
{\left[\begin{array}{ccccc}
\mathbf{K}_{\Gamma}^{1} & \mathbf{0} & \mathbf{0} & \mathbf{0} & { }^{t} \mathcal{B}_{1} \\
\mathbf{0} & {\left[\mathcal{K}^{1}\right]} & \mathbf{0} & 0 & 0 \\
\mathbf{0} & \mathbf{0} & \mathbf{K}_{\Gamma}^{2} & \mathbf{0} & { }^{t} \mathcal{B}_{2} \\
\mathbf{0} & 0 & \mathbf{0} & {\left[\mathcal{K}^{2}\right]} & 0 \\
\mathcal{B}_{1} & 0 & \mathcal{B}_{2} & 0 & 0
\end{array}\right]\left[\begin{array}{c}
\mathbf{u}_{\Gamma}^{1} \\
\mathbf{q}^{1} \\
\mathbf{u}_{\Gamma}^{2} \\
\mathbf{q}^{2} \\
\mathbf{p}
\end{array}\right]} \\
=\omega^{2}\left[\begin{array}{ccccc}
\mathbf{M}_{\Gamma}^{1} & { }^{t} \mathcal{A}_{m}^{1} & \mathbf{0} & \mathbf{0} & \mathbf{0} \\
\mathcal{A}_{m}^{1} & {\left[\mathcal{M}^{1}\right]} & \mathbf{0} & 0 & 0 \\
\mathbf{0} & \mathbf{0} & \mathbf{M}_{\Gamma}^{2} & { }^{t} \mathcal{A}_{m}^{2} & \mathbf{0} \\
\mathbf{0} & 0 & \mathcal{A}_{m}^{2} & {\left[\mathcal{M}^{2}\right]} & 0 \\
\mathbf{0} & 0 & \mathbf{0} & 0 & 0
\end{array}\right]\left[\begin{array}{c}
\mathbf{u}_{\Gamma}^{1} \\
\mathbf{q}^{1} \\
\mathbf{u}_{\Gamma}^{2} \\
\mathbf{q}^{2} \\
\mathbf{p}
\end{array}\right]
\end{array}
$$

Equation (78) is rewritten using the global notation introduced in Eq. (77) and is then similar to Eq. (44). Consequently, we can use the method presented in Section 3.4 for solving this spectral problem.

\subsubsection{General comments}

In the case of a finite element discretization with incompatible mesh on $\Gamma$, the method presented in Section 4.3 (Eqs. (75) and (78)) is efficient because, since $\mathcal{B}_{1}$ and $\mathcal{B}_{2}$ are independent of $\omega$, the SVD is carried out once and for all (even if the sizes of the matrices of the discretized operators $\mathcal{B}_{1}$ and $\mathcal{B}_{2}$ are important).

\section{Conclusion}

Within a general continuum-based approach, we have presented two dynamic substructuring procedures by modal reduction methods in order to calculate the frequency response function of linear damped structures and the eigenmodes of the associated conservative systems. The free-interface and fixed-interface modes of each substructure are used within a mixed variational formulation involving Lagrange multiplier fields defined on the coupling interfaces. Generally, the introduction of a Lagrange multiplier field associated with kinematic linear constraints induces some difficulties for the construction of the solution due to the rank deficiency of the obtained linear system. In the present paper, the Singular Value Decomposition (SVD) method is applied to the frequencyindependent Lagrange multiplier terms. The use of SVD is particularly efficient due to a relatively small number of degrees of freedom in the reduced model and is used once. Therefore, the SVD appears as an efficient and reliable tool for this problem.

\section{References}

Argyris, J., and Meljnek, H.P., 1991, Dynamics of Structures, NortHolland, Amsterdam.

Brezzi, F., and Fortin, M., 1991, Mixed and Hybrid Finite Element Methods, Springer.

Craig Jr., R.R., 1985, "A Review of Time Domain and Frequency Domain Component Mode Synthesis Method,", Combined ExperimentalAnalytical Modeling of Dynamic Structural Systems, edited by Martinez, D.R. and Miller, A.K., ASME-AMD, Vol. 67.

Craig Jr., R.R., and Bampton, M.C.C., 1968, "Coupling of Substructures for Dynamic Analysis," AIAA Journal, Vol. 6., pp. 1313-1319. Dautray R., and Lions J.-L., 1992, Mathematical Analysis and Numerical Methods for Science and Technology, Springer-Verlag, Berlin.

Farhat, C., and Geradin, M., 1994, "On a Component Mode Method and its Application to Incompatible Substructures," Computer and Structures, Vol. 51, No. 5, pp. 459-473.

Farstad, J.E., and Singh, R., 1995, "Structurally Transmitted Dynamic Power in Discretely Joined Damped Component Assemblies", J. Acoust. Soc. Am., Vol. 97, No. 5, pp. 2855-2865.

Flashner, H., 1986, "An Orthogonal Decomposition Approach to Modal Synthesis", Int. J. Num. Meth. Eng., Vol. 23, No. 3, pp. 471-493.

Golub, G.H., and Van Loan, C.F., 1989, Matrix Computations, 2nd Edition, The John Hopkins University Press, Baltimore and London.

Guyan, R.J., 1965, Reduction of Stiffness and Mass Matrices, AIAA Journal, Vol. 3.

Hale, A.L., and Meirovitch, L., 1980, " A General Substructure Synthesis Method for the Dynamic Simulation of Complex Structures", Journal of Sound and Vibration, Vol. 69, No. 2, pp. 309-326.

Hurty, W.C., 1965, "Dynamic Analysis of Structural Systems Using Component Modes," AIAA Journal, Vol. 3, No. 4, pp. 678-685.

Jen, C.W., and Johnson, D.A., and Dubois, F., 1995, "Numerical Modal Analysis of Structures Based on a Revised Substructure Synthesis Approach", Journal of Sound and Vibration, Vol. 180, No. 2, pp. 185203.

Klein, L.R., and Dowell, E.H., 1974, "Analysis of Modal damping by Component Modes Method Using Lagrange Multipliers", Journal of Applied Mechanics, Vol. 39, pp.727-732.

Kree, P., and Soize, C., 1986, Mathematics of Random Phenomena, Reidel, Dordrecht.

Leung, A.Y.T., 1993, Dynamic Stiffness and Substructures, SpringerVerlag, New York.

MacNeal, R.H., 1971, "A Hybrid Method of Component Mode Synthesis," Computers and Structures, Vol. 1, pp. 581-601.

Marsden J.E., and Hughes T.J.R., 1983, Mathematical Foundations of Elasticity, Prentice Hall, Englewood Cliffs, New Jersey. 
Min, K.W., Igusa, T., and Achenbach, J.D., 1992, "Frequency Window Method for Strongly Coupled and Multiply Connected Structural Systems: Multiple-Mode Windows", Journal of Applied Mechanics, Vol. 59, pp.244-252.

Morand, H.J.P., and Ohayon, R.,1995, Fluid Structure Interaction, Wiley, New York.

Rubin, S., 1975, "Improved Component Mode Representation for Structural Dynamic Analysis," AIAA Journal, Vol. 18, No 8, pp. 995-1006.

Rook, T.E., and Singh, R., 1995, "Power Flow Through Multidimensional Compliant Joints Using Mobility and Modal Approaches", J. Acoust. Soc. Am., Vol. 97, No. 5, pp. 2882-2891.

Schmidt, Th., and Müller, P.C., 1993, "A Parameter Estimation Method for Multibody Systems with Constraints," Advanced Multibody System Dynamics, Edited by W. Schiehlen, Kluwer Academic Publishers, Dordrecht/Boston/London, pp. 427-433.

Shabana, A.A., 1991, "Constrained Motion of Deformable Bodies," Int. J. Num. Meth. Eng., Vol. 32, pp. 1813-1831.

Singh, R.P., and Likins, P.W., 1985, "Singular Value Decomposition for Constrained Dynamical Systems," Journal of Applied Mechanics, Vol. 52, pp. 943-948.

Soize, C., Desanti, A., and David, J.M., 1992, "Numerical Methods in Elastoacoustic for Low and Medium Frequency Ranges," La Recherche Aérospatiale (English Edition), Vol. 5, pp. 25-44. 